\title{
FrequentNet: A Novel Interpretable Deep Learning Model for Image Classification
}

\author{
Yifei $\mathrm{Li}^{1}$, Kuangyan Song ${ }^{2}$, Yiming $\mathrm{Sun}^{3 \mathrm{a}}$, and Liao Zhu ${ }^{4} \oplus^{\mathrm{b}}$ \\ ${ }^{1}$ Department of Computer Science, Zhejiang University, China \\ ${ }^{2}$ Microsoft \\ ${ }^{3}$ Instacart \\ ${ }^{4}$ Department of Statistics and Data Science, Cornell University, Ithaca, USA \\ lyeeef@gmail.com,nachtsky@gmail.com,sunstat.faust@gmail.com,lz384@cornell.edu
}

\begin{abstract}
Keywords: Neural Network, Machine Learning, Wavelet Analysis, Fourier Analysis
Abstract: This paper has proposed a new baseline deep learning model of more benefits for image classification. Different from the convolutional neural network $(\mathrm{CNN})$ practice where filters are trained by back propagation to represent different patterns of an image, we are inspired by a method called "PCANet" (Chan et al.(2015)Chan, Jia, Gao, Lu, Zeng, and Ma) to choose filter vectors from basis vectors in frequency domain like Fourier coefficients or wavelets without back propagation. Researchers have demonstrated that those basis in frequency domain can usually provide physical insights, which adds to the interpretability of the model by analyzing the frequencies selected. Besides, the training process will also be more time efficient, mathematically clear and interpretable compared with the "black-box" training process of CNN.
\end{abstract}

\section{Introduction}

Convolutional Neural Networks(CNN) (Jarrett et al.(2009)Jarrett, Kavukcuoglu, Ranzato, and LeCun) has witnessed tremendous success in image classification (Krizhevsky et al.(2012)Krizhevsky, Sutskever, and Hinton), with filters performing convolution operations that aim to capture different patterns in an image. Yet in order to obtain these filter vectors, it is necessary to solve the complicated optimization problem of resorting to back propagation, which makes the whole process a black-box, thereby leading to the lack of clear mathematical interpretations of the resulting filter vectors. (Chan et al.(2015)Chan, Jia, Gao, Lu, Zeng, and Ma) put forward a baseline model for image classification, which does not require any kind of back propagation to learn those filters. Instead, they suggested adopting left eigen-vectors of stacked images which are commonly known as principal component vectors as the candidate filters. This idea stems from the eigen-decomposition where we can decompose the target onto the orthogonal basis (eigen-vectors). Projection along each orthogonal basis can represent

\footnotetext{
${ }^{\mathrm{a} C}$ Corresponding author.

b (iD) https://orcid.org/0000-0001-7261-1773
}

"non-overlapping" patterns in the image. However, the process of obtaining those eigen-vectors can be quite time-consuming, especially for large datasets, even when some randomized algorithms (Halko et al.(2011)Halko, Martinsson, and Tropp) are applied. In the classical literature regarding computer vision, researchers have developed multi-scaled representation of images without resorting to optimization. Two most widely used ones are Discrete Fourier Transformation(DFT) (Nordberg(1995)) and Wavelets analysis (Mallat(1996)).

Researchers have found that different frequencies can capture different levels of information in images. For example, the high-pass filter will only select high-frequency signals to get the structured information like edges, while the low-pass filter will select low-frequency signals and thus generate an over-smoothed and blurry image. (Costen et al.(1996)Costen, Parker, and Craw). There are many traditional models focusing on the detection of high-frequency information. For instance, typical gradient-based methods such as the sobel operator (Gao et al.(2010)Gao, Zhang, Yang, and Liu), prewitt operator (Yang et al.(2011)Yang, Wu, Zhao, Li, and Zhail) and canny operator (Canny(1986)) detect the high-frequency information in the 1-order gradi- 
ent domain. The laplacian operator (Wang(2007)) focuses on the 2-order gradient, which has been widely applied in image processing to sharpen images. We refer (Kumar et al.(2013)Kumar, Saxena, et al.) for interested readers to gain a comprehensive picture of edge detectors in image processing. In this work, our major focus is the discrete Fourier transformation, since it has a simpler form and can be easily extended to convolutional filters.

In this paper, we shall explore the possibilities of adopting basis from DFT and Wavelets analysis as candidates for filter vectors. Before presenting our algorithms, let us have a brief review of both Discrete Fourier Transformation and Wavelets analysis.

\subsection{Discrete Fourier Transformation}

Discrete Fourier Transformation (DFT) (Beerends et al.(2003)Beerends, ter Morsche, Van den Berg, and Van de Vrie) can represent vectorized images with different components at different frequencies. Mathematically, given a vectorized image vector $\mathbf{x}$ of length $n$, 1D DFT decompose $\mathbf{x}$ into Fourier basis $\mathbf{e}^{i \omega_{k}}=$ $\mathbf{c}\left(\omega_{k}\right)-i \mathbf{s}\left(\omega_{k}\right)$ with coefficient as the inner product between $\mathbf{x}$ and $\mathbf{e}^{i \omega_{k}}$. Here $\mathbf{s}_{k}, \mathbf{c}_{k}$ are defined as

$$
\begin{aligned}
& \mathbf{c}\left(\omega_{k}\right)=\frac{1}{\sqrt{n}}\left(1, \cos \omega_{k}, \ldots, \cos (n-1) \omega_{k}\right)^{\top}, \\
& \mathbf{s}\left(\omega_{k}\right)=\frac{1}{\sqrt{n}}\left(1, \sin \omega_{k}, \ldots, \sin (n-1) \omega_{k}\right)^{\top},
\end{aligned}
$$

and $\omega_{k}=\frac{2 \pi k}{n}$ are the discrete Fourier frequencies whose index $k$ belongs to a set denoted as $F_{n}$ : $\left\{-\left[\frac{n-1}{2}\right], \ldots,\left[\frac{n}{2}\right]\right\}$ where $[x]$ is the integer part of $x$. Noticing $\left\{s_{k}, c_{k}, k \in F_{n}\right.$ and $\left.k \geq 0\right\}$ forms a complete orthogonal basis for $\mathbb{R}^{n}\left(\mathbf{s}_{0}=\mathbf{0}\right)$, sometimes researchers only consider the non-negative frequencies in the $F_{n}$. In the following parts, the set containing all non-negative indices in $F_{n}$ is referred to as $F_{n}^{+}$.

\subsection{Wavelets Analysis}

Different from DFT, wavelets aim to conduct spectral analysis locally in the graph which can be seen from the difference in their orthogonal basis: each Fourier coefficient vector share while wavelets basis vector, which will be introduced later, behaves more abruptly (Strang(1993)), (Chui(2016)), (Daubechies(1992)), (Mallat(1988)). This means wavelets can captures edge information in the computer vision compared to DFT, which will be demonstrated in recover image in Figure6 In this paper we chosee to apply Daubechies D4 Wavelet Transform to the image. The wavelet and scaling function coefficients of the Daubechies
D4 wavelet are

$$
\begin{aligned}
& \mathbf{h}=\left[\frac{1+\sqrt{3}}{4}, \frac{3+\sqrt{3}}{4}, \frac{3-\sqrt{3}}{4}, \frac{1-\sqrt{3}}{4}\right] \\
& \mathbf{g}=\left[\frac{1-\sqrt{3}}{4}, \frac{\sqrt{3}-3}{4}, \frac{3+\sqrt{3}}{4}, \frac{-1-\sqrt{3}}{4}\right] .
\end{aligned}
$$

where $\mathbf{h}$ is the scaling function coefficients and $\mathbf{g}$ is the wavelet function coefficients. $\mathbf{h}$ is like calculating the moving average, which performs as low pass filtering, while $\mathbf{g}$ is capturing the comparison of local graph performing as high pass filtering in the above section. Then for vectorized image $\mathbf{x}$, the first layer wavelet transform is like linear transformation in for $\mathrm{n}$ filter vectors as

$$
\begin{aligned}
& {\left[\begin{array}{ccccccc}
h_{0} & h_{1} & h_{2} & h_{3} & \cdots & \cdots & \cdots \\
0 & 0 & h_{0} & h_{1} & h_{2} & h_{3} & \cdots \\
\vdots & \vdots & \vdots & \vdots & \vdots & \vdots & \ddots
\end{array}\right] \mathbf{x} .} \\
& {\left[\begin{array}{ccccccc}
g_{0} & g_{1} & g_{2} & g_{3} & \cdots & \cdots & \cdots \\
0 & 0 & g_{0} & g_{1} & g_{2} & g_{3} & \cdots \\
\vdots & \vdots & \vdots & \vdots & \vdots & \vdots & \ddots
\end{array}\right] \mathbf{x} .}
\end{aligned}
$$

where the first half of the transform is computed via (3) and the second half of the transform via (4). The second layer wavelet transform is then computed by taking the first half of the first layer transform results, which is results of (3), treat it as an averaged version of the original vectorized image $\mathbf{x}$, and perform linear transformation using row vectors in (3) and (4) with length $\frac{n}{2}$. Then each successive iteration simply repeat the same process. Notice the vector $\mathbf{x}$ and the averaged $\mathbf{x}$ in each layer will be padded to even length if it has an odd length. The final level transformation results and the second half results from the previous levels, which are the results of (4) of the previous levels, will be concatenated to form the wavelets transformation results. The structure for level-3 wavelet transform is shown in 1. In this case, we can treat each line of left matrix in (3) and (4) as the pool of our potential filter vectors.

\section{FrequentNet}

Our method is inspired by (Chan et al.(2015)Chan, Jia, Gao, Lu, Zeng, and Ma), with the difference of adopting basis in frequency domain rather than principal component vectors. The overall pipeline is the same as PCANet, which are composed of two procedures. The first procedure is selecting filter vectors, performing convolutional/filter transformation and repeating it once again. (Implementing this procedure 


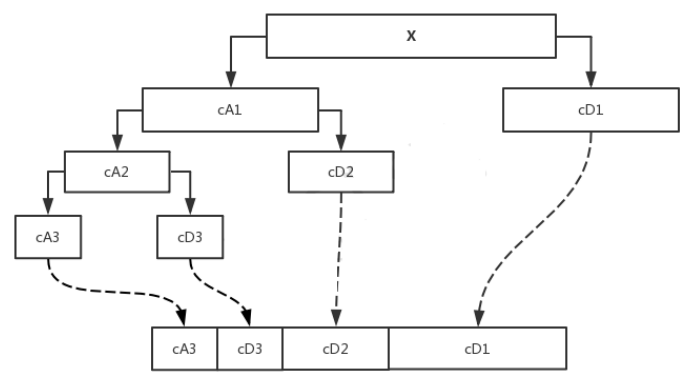

Figure 1: The level-3 wavelet transformation: the first half of the wavelet transformation $\left(\mathbf{c A}_{\mathbf{1}}\right.$ and $\mathbf{c} \mathbf{A}_{\mathbf{2}}$ ) will be taken to perform the next level transformation, and then concatenate the final level transformation $\left(\mathbf{c A}_{\mathbf{3}}\right.$ and $\mathbf{C D}_{\mathbf{3}}$ ) results with the latter half of transformation in the previous levels $\left(\mathbf{C D}_{\mathbf{1}}\right.$ and $\mathbf{c D}_{2}$ ) as the level-3 wavelet transformation result.

once again and presenting its results have also been taken into account). The second procedure is applying hashing and histogram and running the support vector machine to output from the previous procedure and achieve classification.

\subsection{Problem Setup}

We mainly follow the settings in (Chan et al.(2015)Chan, Jia, Gao, Lu, Zeng, and Ma). Provided with $N$ input training images: $\left\{I_{i}\right\}_{i=1}^{N}$ of size $m \times n$, we set the patch size (or $2 \mathrm{D}$ filter size) as $k_{1} \times k_{2}$. Throughout the paper, padding size has been set to be $\left(k_{1}-1\right) / 2$ for the top and the bottom, $\left(k_{2}-1\right) / 2$ for the left and the right, with the padding value at zero. Meanwhile, stride for patch is set to be one, which is also listed in Table 3. Under this setting after the filter transformation, the output size will be the same as the input image size: $m \times n$. We call those vectorized patches $\mathbf{x}_{i, 1}, \cdots, \mathbf{x}_{i, m n}$ where the first index is for images and the second index is for patches. The patch mean has been subtracted from each patch and stacked for each image as

$$
\overline{\mathbf{X}}_{i}=\left[\overline{\mathbf{x}}_{i, 1}, \cdots, \overline{\mathbf{x}}_{i, j}, \cdots, \overline{\mathbf{x}}_{i, m n}\right], 1 \leq j \leq m n
$$

where $\overline{\mathbf{x}}_{i, j}$ is the de-meaned patch. We further stack them as $\overrightarrow{\mathbf{X}}_{i}$ again to obtain

$$
\overline{\mathbf{X}}=\left[\overline{\mathbf{X}}_{1}, \cdots, \overline{\mathbf{X}}_{i}, \cdots, \overline{\mathbf{X}}_{N}\right] \in \mathbb{R}^{k_{1} k_{2} \times N m n}, 1 \leq i \leq N
$$

Filter vectors are intended for representing patterns in columns in $\mathbf{x}_{i, j}$ effectively. PCANet chooses the filter vectors to be the top left eigen-vectors of $\overline{\mathbf{X}}$. In this paper, adopting basis in DFT and wavelets have been proposed in a detailed manner.

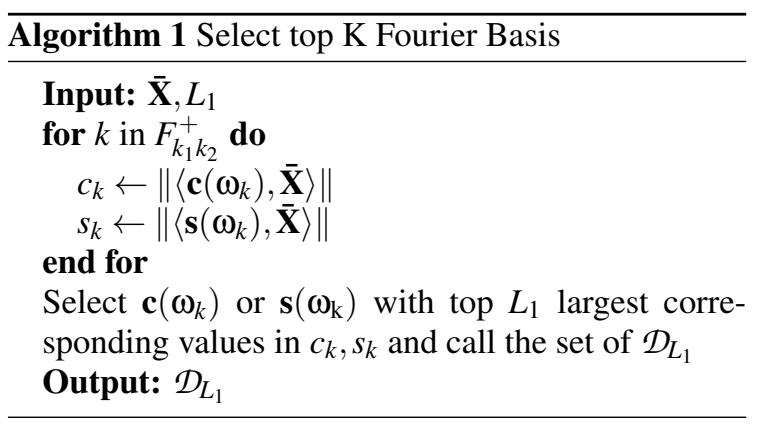

\subsection{FourierNet}

The First Stage: We choose $\left\{\mathbf{c}\left(\omega_{k}\right), \mathbf{s}\left(\omega_{k}\right)\right\}, k \in F_{n}^{+}$ as our candidate orthogonal basis, and then select filters at different frequencies based on the magnitude of the inner product of vectorized patches $\mathbf{x}_{i, j}$ and candidate filters, as summarized in Algorithm 1 . With the obtained $L_{1}$ filters $\mathbf{v}_{1}, \cdots, \mathbf{v}_{k}, \cdots, \mathbf{v}_{L_{1}}$, every input image $I_{i}$ is mapped to $L_{1}$ new feature maps:

$$
I_{i}^{\ell}=I_{i} * \operatorname{mat}_{\mathrm{k}_{1}, \mathrm{k}_{2}}\left(\mathbf{v}_{\ell}\right),
$$

where $*$ is the two dimensional convolution and mat is an operator reshaping filter back to its original shape of the patch: $k_{1} \times k_{2}$. For convenience, we index the vector set $\mathbf{v} \in \mathcal{D}_{L_{1}}$ based on $\|\langle\mathbf{v}, \overline{\mathbf{X}}\rangle\|$ reversely, i.e., $\left\|\left\langle\mathbf{v}_{1}, \overline{\mathbf{X}}\right\rangle\right\| \geq \cdots \geq\left\|\left\langle\mathbf{v}_{L_{1}}, \overline{\mathbf{X}}\right\rangle\right\|$.

The Second Stage: After the first stage, for each filter vector $\mathbf{v}_{\ell}$ in $\mathcal{D}_{L_{1}}$, we can get a new set of feature matrices of the same size as the original image: $m \times n$. For the new $L_{1} N$ feature matrices denoted by $I_{i}^{\ell} \in$ $\mathbb{R}^{m \times n}, i=1, \cdots, N, \ell=1, \cdots L_{1}$, steps in the first stage are repeated to continue to stack all the overlapping patches and subtract mean from them. For each filter $\mathbf{v}_{\ell}, 1 \leq \ell \leq L_{1}$, by stacking Nmn de-meaned patches we can get $\overline{\mathbf{Y}}^{\ell}$, like $\overline{\mathbf{X}}$ defined in the first stage:

$$
\overline{\mathbf{Y}}^{\ell}=\left[\overline{\mathbf{y}}_{i, 1}^{\ell} ; \cdots ; \overline{\mathbf{y}}_{i, m n}^{\ell}\right] \in \mathbb{R}^{k_{1} k_{2} \times N m n}, 1 \leq i \leq N
$$

where $\overline{\mathbf{y}}_{i, j}^{\ell}$ is the de-meaned patch in the $I_{i}$. Then we stack all $\overline{\mathbf{Y}}^{\ell}$ to obtain our final feature matrix

$$
\overline{\mathbf{Y}}=\left[\overline{\mathbf{Y}}^{1} ; \cdots ; \overline{\mathbf{Y}}^{\ell} \cdots ; \overline{\mathbf{Y}}^{L 1}\right] \in \mathbb{R}^{k_{1} k_{2} \times L_{1} N m n} .
$$

Algorithm 1 is applied on $\overline{\mathbf{Y}}$ to select top $L_{2}$ Fourier basis. Following the above definition, we call selected basis $\mathbf{u}_{1}, \cdots, \mathbf{u}_{L_{2}}$. According to our setting in stride and padding, the output feature matrix is still of the same size as the original input image: $m \times n$. We call it the output feature matrix $O_{i}^{\ell}$ :

$$
O_{i}^{\ell_{2}}=I_{i}^{\ell_{1}} * \text { mat }_{\mathrm{k}_{1}, \mathrm{k}_{2}}\left(\mathbf{u}_{\ell_{2}}\right), 1 \leq \ell_{1} \leq \mathrm{L}_{1}, 1 \leq \ell_{2} \leq \mathrm{L}_{2} .
$$


Output Stage: In this section, we follow exactly the procedure of output stage in (Chan et al.(2015)Chan, Jia, Gao, Lu, Zeng, and Ma) to finally transform the output of feature matrix, and run support vector machine to make the prediction. Steps have been briefly sketched. For more details, we refer readers to Chan et al.(2015)Chan, Jia, Gao, Lu, Zeng, and Ma). This procedure works for both the first and second stages, with notations used in the two stage model for explanation. Given the $N L_{1}$ input feature matrix: $I_{i}^{\ell_{1}}$ and selected $L_{2}$ filters $\mathbf{u}_{\ell_{2}}$, we aggregate filter information as

$$
\mathcal{T}_{i}^{\ell_{1}}=\Sigma_{\ell_{2}=1}^{L_{2}} 2^{\ell_{2}-1} H\left(I_{i}^{\ell_{1}} * \mathbf{u}_{\ell_{2}}\right)
$$

where $\mathrm{H}$ is binary operator turning positive elements to be one while others to zero element wise. It is easy to see each element fall into $\left[0,2^{L_{2}}-1\right]$.

For each patch in $\mathcal{T}_{i}^{\ell_{1}}$, we computer the histogram ( $2^{L_{2}}$ bins) with two parameters called block size and block stride. Concatenating all histograms for each $\mathcal{T}_{i}^{\ell_{1}}$ of Bhist $\left(\mathcal{T}_{i}^{\ell_{1}}\right)$ across $L_{1}$ filters, we can obtain the feature vector

$$
\mathbf{f}_{i}=\left[\operatorname{Bhist}\left(\mathcal{T}_{\mathrm{i}}^{1}\right), \cdots, \operatorname{Bhist}\left(\mathcal{T}_{\mathrm{i}}^{\mathrm{L}_{1}}\right)\right] \in \mathbb{R}^{2^{\left(2^{\left.L_{2}\right)} L_{1} B\right.}} .
$$

Then we run support vector machine on feature vector $\mathbf{f}_{i}$ to obtain the classification results.

\subsection{WaveletsNet}

For WaveletsNet, the whole process is the same as FourierNet except that now the pool of candidate filter vectors become all rows in orthogonal basis in wavelets. In this paper, we only consider basis from three layers in Daubechies D4 wavelets. Again, for stage I, $L_{1}$ filter vectors are selected based on the magnitude of inner product between filter vectors and vectorized image vectors. At this point, we can either go to the output stage, or repeat the selecting procedure to further select $L_{2}$ filters and then move on to the output stage.

\subsection{PCANet, RandNet}

In (Chan et al.(2015)Chan, Jia, Gao, Lu, Zeng, and Ma), researchers proposed PCANet where the filter candidates are from PCA vectors for $\overline{\mathbf{X}}$ in the first stage and for $\overline{\mathbf{Y}}$ in the second stage. With the importance of PCA vectors naturally sorted by corresponding eigen-values, we just choose the top $L_{1}$ or $L_{2}$ PCA vectors. For RandNet, as the name suggests, filter vectors are chosen randomly from multivariate Gaussian distributions. By comparing our method with those two methods, it is worth noting that unlike PCANet, filters in FreqNet are data independent.
Table 1: Model Structure Description

\begin{tabular}{ll}
\hline Model & Description \\
\hline FourierNet-1 & 1-stage FourierNet \\
FourierNet-2 & 2-stage FourierNet \\
WaveletsNet-1 & 1-stage FourierNet \\
WaveletsNet-2 & 2-stage FourierNet \\
PCANet-1 & 1-stage PCANet \\
PCANet-2 & 2-stage PCANet \\
RandNet-1 & 1-stage RandNet \\
RandNet-2 & 2-stage RandNet \\
FourierNet2D-2 & 2-stage FourierNet (2D Fourier basis) \\
\hline
\end{tabular}

Table 2: Descriptions of MNIST variations picked for experiment

\begin{tabular}{ll}
\hline Dataset & Description \\
\hline basic & A smaller subset of standard MNIST \\
bg-rand & MNIST with noise background \\
rot & MNIST with rotation \\
bg-img & MNIST with image background \\
bg-img-rot & MNIST with rotation and image background \\
\hline
\end{tabular}

\section{Experiments}

We evaluated the performances of proposed models, which are described in the Table 11, and compared with PCANet and RandNet on two tasks, the hand-written digits recognition and object recognition. Then we analyzed the extracted filters in FourierNet and WaveletsNet by visualizing the filtered image by selected filters. We use level-1 Daubechies D4 wavelet transformation for all kinds of WaveletsNet below during the experiments. The code for the model and experiments are publicly available ${ }^{1}$.

\subsection{Hand-written Digits Recognition}

We first conducted the experiment on MNIST variations. The MNIST (LeCun et al.(1998)LeCun, Bottou, Bengio, Haffner, et al.) and MNIST variations (Larochelle et al.(2007)Larochelle, Erhan, Courville, Bergstra, and Bengio) are common benchmarks for testing hierarchical representations (Chan et al.(2015)Chan, Jia, Gao, Lu, Zeng, and Ma). We pick a subset of MNIST variations to experiment on. The datasets and their descriptions are listed in the Table 2.

\subsubsection{Experiment Setup}

Following the experiment setup in (Chan et al.(2015)Chan, Jia, Gao, Lu, Zeng, and Ma), we conducted experiments using both one-stage and two-stage models. For one-stage model, we fixed

\footnotetext{
${ }_{1}^{1}$ https://github.com/ijcaiworkshop2020/freqnet
} 
Table 3: Experiment setup of one-stage models

\begin{tabular}{lllcl}
\hline Dataset & $L_{1}$ & patch size & $\begin{array}{c}\text { block } \\
\text { size }\end{array}$ & $\begin{array}{l}\text { block } \\
\text { stride }\end{array}$ \\
\hline basic & 8 & $7 \times 7$ & $7 \times 7$ & 3 \\
bg-rand & 8 & $7 \times 7$ & $4 \times 4$ & 2 \\
rot & 8 & $7 \times 7$ & $4 \times 4$ & 2 \\
bg-img & 8 & $7 \times 7$ & $4 \times 4$ & 2 \\
bg-img-rot & 8 & $7 \times 7$ & $4 \times 4$ & 2 \\
\hline
\end{tabular}

Table 4: Experiment setup of two-stage models

\begin{tabular}{llllll}
\hline Dataset & $L_{1}$ & $\begin{array}{c}\text { patch } \\
\text { size }\end{array}$ & $L_{2}$ & $\begin{array}{c}\text { block } \\
\text { size }\end{array}$ & $\begin{array}{l}\text { block } \\
\text { stride }\end{array}$ \\
\hline basic & 6 & 8 & $7 \times 7$ & $7 \times 7$ & 3 \\
bg-rot & 6 & 8 & $7 \times 7$ & $4 \times 4$ & 2 \\
bg-rand & 6 & 8 & $7 \times 7$ & $4 \times 4$ & 2 \\
bg-img & 6 & 8 & $7 \times 7$ & $4 \times 4$ & 2 \\
bg-img-rot & 6 & 8 & $7 \times 7$ & $4 \times 4$ & 2 \\
\hline
\end{tabular}

patch size to $7 \times 7$, then investigated the impact of number of filters $L_{1}$. For two-stage models, we followed the recommended configurations for different datasets in the original PCANet paper. Notice instead of using the parameter block overlap ratio in the block-wise histogram stage, we explicitly define the block stride by computing the overlap and truncated to the nearest integer. For all the experiments, we fixed patch stride to 1 and padded zero around the image in the patch collection stage. Other detailed experiment parameters, for both one-stage and two-stage models, are listed in Table 3 and Table 4 respectively.

\subsubsection{Experiment Results}

The test accuracy of the one stage models on the selected datasets, with the number of filters varies from 2 to 8 are shown in Figure 3 We can see from the results that the test accuracy increases when the number of filters grows. The test results for the two-stage models, with the key setup in Table 4 are listed in Table 5 One can see that FourierNet-2 and WaveletsNet-2 achieve similar testing accuracy on these datasets.

\subsubsection{Discussion}

To understand what the proposed models are learning, we listed the learned first and second stage Fourier filters from $b$-rand dataset in Figure 2. We could see from the visualized filters that most of them are on the low frequency side, like the first four columns of Figure 2. Furthermore, we investigated the selected frequencies for other MNIST variations, and
Table 5: Testing Accuracies(\%) of different models of MNIST variations. The model parameters follow Table 3 and Table 4

\begin{tabular}{llllll}
\hline Model & basic & $\begin{array}{c}\text { bg- } \\
\text { rand }\end{array}$ & rot & $\begin{array}{l}\text { bg- } \\
\text { img }\end{array}$ & $\begin{array}{c}\text { bg- } \\
\text { img-rot }\end{array}$ \\
\hline FourierNet-2 & 98.05 & 90.50 & 89.45 & 86.55 & 60.25 \\
FourierNet-1 & 98.75 & 89.95 & 85.15 & 85.45 & 49.65 \\
WaveletsNet-2 & 98.55 & 88.05 & 83.60 & 84.45 & 49.25 \\
WaveletsNet-1 & 97.55 & 83.60 & 83.60 & 78.05 & 42.20 \\
PCANet-2 & 98.15 & 91.55 & 89.50 & 87.20 & 62.50 \\
PCANet-1 & 98.65 & 91.80 & 87.35 & 86.65 & 54.35 \\
RandNet-2 & 97.55 & 82.70 & 86.00 & 83.40 & 40.00 \\
RandNet-1 & 97.95 & 70.90 & 77.55 & 67.55 & 29.90 \\
\hline
\end{tabular}

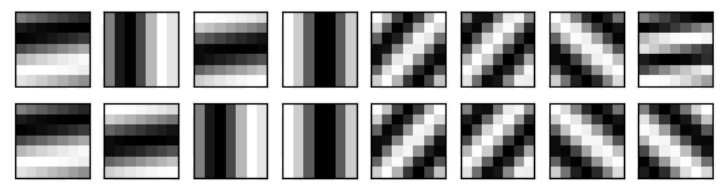

Figure 2: The Fourier filters learned from $b g$-rand dataset. Top: the first stage filters. Bottom: the second stage filters.

we observe that the low frequencies, such as $2 \pi / 49$, $12 \pi / 49$ and $14 \pi / 49$ appear in selected frequencies for all the datasets. Hence, we believe that the low frequency components carry significant information for the MNIST variations. We report the selected Fourier basis in FourierNet-2 for MNIST variations in Table 6.

In order to get an intuitive sense of the features captured by the learned filters, we visualized some of the convolution results of an original image from dataset and a single filter selected by the models. Two samples are selected randomly from MNIST dataset, and are convoluted with learned filters, including Fourier filter, Wavelet filter, PCA filter and Random filter. The feature captured by the learned filters are shown in Figure 6, each of the images is the convolution results of a raw image sample with a selected filter.

\subsubsection{Extension}

We further constructed FourierNet2D, which is a twostage FourierNet using 2D Fourier basis, and tested on MNIST variations. The results are shown in Table 7. However, we did not observe improvements in test accuracy using 2D Fourier basis.

Some related research can be found in (Zhu et al.(2021c)Zhu, Wu, and Wells) (Zhu et al.(2020)Zhu, Basu, Jarrow, and Wells) (Zhu(2020)) (Zhu et al.(2021a)Zhu, Jarrow, and Wells) (Zhu et al.(2021b)Zhu, Sun, and Wells) (Jarrow et al.(2021)Jarrow, Murataj, Wells, and Zhu) (Sun et al.(2021)Sun, Guo, Tropp, and Udell) (Sun 


\begin{tabular}{|c|c|c|c|c|c|c|c|c|c|c|c|c|c|c|}
\hline Dataset & \multicolumn{6}{|c|}{ Selected first stage basis } & \multicolumn{8}{|c|}{ Selected second stage basis } \\
\hline \multirow{3}{*}{$\begin{array}{c}\text { basic } \\
\text { bg-rand } \\
\text { rot } \\
\text { bg-img } \\
\text { bg-img-rot }\end{array}$} & $\mathbf{c}\left(\omega_{1}\right)$ & $\mathbf{c}\left(\omega_{6}\right)$ & $\mathbf{c}\left(\omega_{7}\right)$ & $\mathbf{s}\left(\omega_{1}\right)$ & $\mathbf{s}\left(\omega_{6}\right)$ & $\mathbf{s}\left(\omega_{7}\right)$ & $\mathbf{c}\left(\omega_{1}\right)$ & $\mathbf{c}\left(\omega_{6}\right)$ & $\mathbf{c}\left(\omega_{7}\right)$ & $\mathbf{c}\left(\omega_{8}\right)$ & $\mathbf{s}\left(\omega_{1}\right)$ & $\mathbf{s}\left(\omega_{6}\right)$ & $\mathbf{s}\left(\omega_{7}\right)$ & $\mathbf{s}\left(\omega_{8}\right)$ \\
\hline & $\mathbf{c}\left(\omega_{1}\right)$ & $\mathbf{c}\left(\omega_{6}\right)$ & $\mathbf{c}\left(\omega_{7}\right)$ & $\mathbf{s}\left(\omega_{1}\right)$ & $\mathbf{s}\left(\omega_{6}\right)$ & $\mathbf{s}\left(\omega_{7}\right)$ & $\mathbf{c}\left(\omega_{1}\right)$ & $\mathbf{c}\left(\omega_{6}\right)$ & $\mathbf{c}\left(\omega_{7}\right)$ & $\mathbf{c}\left(\omega_{8}\right)$ & $\mathbf{s}\left(\omega_{1}\right)$ & $\mathbf{s}\left(\omega_{6}\right)$ & $\mathbf{s}\left(\omega_{7}\right)$ & $\mathbf{s}\left(\omega_{8}\right)$ \\
\hline & $\mathbf{c}\left(\omega_{1}\right)$ & $\mathbf{c}\left(\omega_{6}\right)$ & $\mathbf{c}\left(\omega_{7}\right)$ & $\mathbf{s}\left(\omega_{1}\right)$ & $\mathbf{s}\left(\omega_{6}\right)$ & $\mathbf{s}\left(\omega_{7}\right)$ & $\mathbf{c}\left(\omega_{1}\right)$ & $\mathbf{c}\left(\omega_{6}\right)$ & $\mathbf{c}\left(\omega_{7}\right)$ & $\mathbf{c}\left(\omega_{9}\right)$ & $\mathbf{s}\left(\omega_{1}\right)$ & $\mathbf{s}\left(\omega_{6}\right)$ & $\mathbf{s}\left(\omega_{7}\right)$ & $\mathbf{s}\left(\omega_{9}\right)$ \\
\hline
\end{tabular}

Table 6: Selected Fourier basis in FourierNet-2 for different datasets. Following the definition in Section $|1.1| \mathbf{c}\left(\omega_{k}\right)$ and $\mathbf{s}\left(\omega_{k}\right)$ are the orthogonal Fourier basis, where $\omega_{k}=\frac{2 k \pi}{49}$ since the patch size for all the MNIST variation datasets is $49(7 \times 7)$. The model parameters follow Table 4

Table 7: Testing Accuracies(\%) of FourierNet2D-2

\begin{tabular}{llllll}
\hline Model & basic & $\begin{array}{c}\text { bg- } \\
\text { rand }\end{array}$ & rot & $\begin{array}{l}\text { bg- } \\
\text { img }\end{array}$ & $\begin{array}{c}\text { bg- } \\
\text { img-rot }\end{array}$ \\
\hline FourierNet2D-2 & 97.80 & 89.60 & 87.60 & 86.05 & 45.30 \\
\hline
\end{tabular}
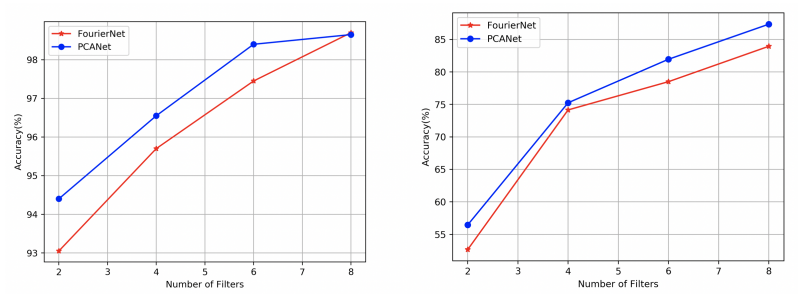

Figure 3: Test accuracy(\%) of FourierNet-1 and PCANet1 on MNIST basic and rot test set for varying number of filters $\left(L_{1}\right)$. We tested $L_{1}$ varies from 2 to 8 .

\section{et al.(2020)Sun, Guo, Luo, Tropp, and Udell).}

\subsection{CIFAR10 Object Recognition}

\subsubsection{Experiment Results}

CIFAR10 contains 10 classes with 50000 training samples and 10000 test samples, which vary in object position, scale, colors and textures (Chan et al.(2015)Chan, Jia, Gao, Lu, Zeng, and Ma). We fix the number of filters in the first stage to 40 , the number of filters in second stage to 5 . We also set the patch size to $5 \times 5$, block size to $8 \times 8$ and block stride to 4. Apart from the two-stage FourierNet and two-stage WaveletsNet, we tried combining Fourier basis and Wavelets basis together to form two combined two-stage models, namely Fourier-PCA, which uses Fourier filters in the first stage and PCA filters for the second stage, and PCA-Fourier, which uses PCA filters in the first stage and Fourier filters in the second stage. The test accuracy of different combinations are listed in Table 8 .

\subsubsection{Discussion}

We now look at the learned filters of FourierNet-2 and PCANet-2 from CIFAR10 dataset, which are shown
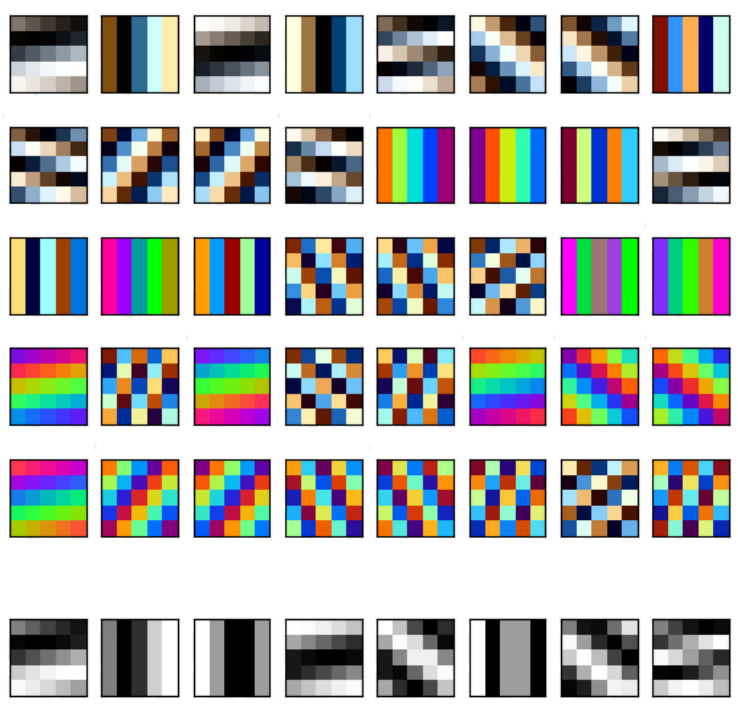

Figure 4: The Fourier filters learned from CIFARIO dataset. Top: the first stage filters, the number of filters for each channel is set to 40 . Bottom: the second stage filters, the number of filters is set to 8 .

Table 8: Testing accuracy(\%) of two-stage models on CIFAR10

\begin{tabular}{ll}
\hline Model & Accuracy \\
\hline FourierNet-2 & 67.70 \\
PCANet-2 & 70.95 \\
Fourier-PCA & 68.30 \\
PCA-Fourier & 69.75 \\
\hline
\end{tabular}

in Figure 4 and Figure 5 respectively. One could easily tell that the first stage filters of FourierNet-2 includes both low frequency and high frequency components, and the second stage filters consist of mainly low frequency filters, while one can hardly get intuitive sense from the visualization of PCANet- 2 filters learned from CIFAR10 dataset. 

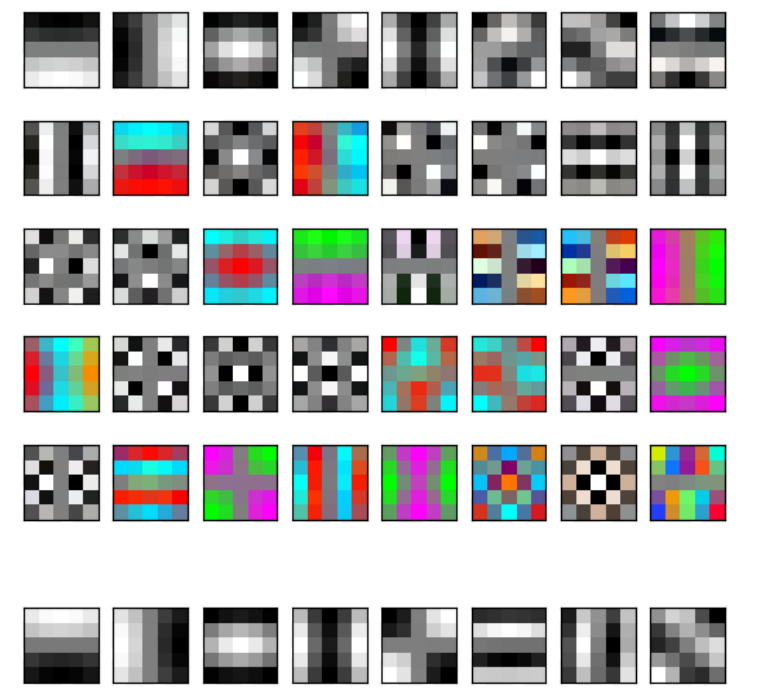

Figure 5: The PCA filters learned from CIFAR10 dataset. Top: the first stage filters, the number of filters for each channel is set to 40 . Bottom: the second stage filters, the number of filters is set to 8 .

\section{Conclusion}

In this paper, we propose adopting orthogonal basis in frequent domain like from Discrete Fourier Transformation or wavelets analysis as candidates for filter vectors in CNN. Different from Chan et al.(2015)Chan, Jia, Gao, Lu, Zeng, and Ma), our filter vectors are data independent, with no requirement for solving any optimization problem in the selection procedure, thereby rendering the whole process more transparent and understandable. Through extensive experiments, it is demonstrated that our method has witnessed comparable results in several benchmark data sets. Furthermore, analysis in frequency domain for computer vision can provide us with insights from different perspectives that cannot be achieved by principal component analysis. In the future, we plan to explore more datasets so as to figure out scenarios where these methods fit best and give full play to their effectiveness.
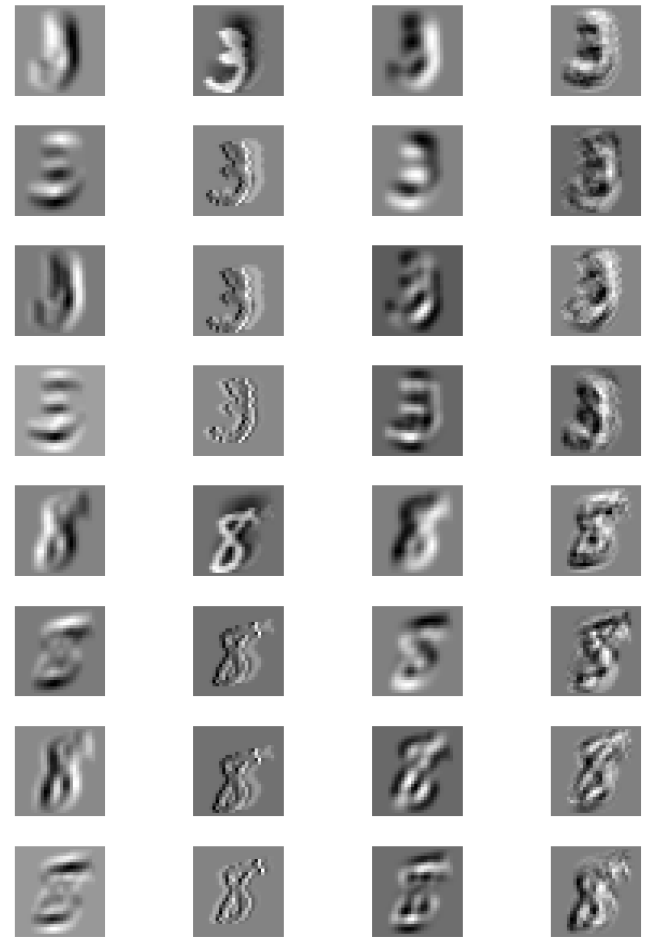

(a) Fourier
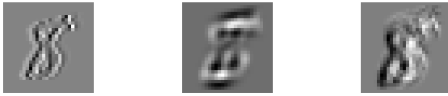
Figure 6: The low rank approximations using a single filter learned from the network, from left to right: (a) Fourier filter; (b) Wavelet filter (c) PCA filter; (d) Random filter. We picked four selected filters for each basis. Then, for each of the selected filters, we performed convolution with the two randomly selected $28 \times 28$ image samples. From the above figures, one could see that Fourier filters and PCA filters extract features with similar patterns, and selected wavelet filters extract "edge-like" features from the images. 


\section{REFERENCES}

René Johan Beerends, Henricus G ter Morsche, JC Van den Berg, and EM Van de Vrie. Fourier and laplace transforms. Fourier and Laplace Transforms, by RJ Beerends and HG ter Morsche and JC van den Berg and EM van de Vrie, pp. 458. ISBN 0521534410. Cambridge, UK: Cambridge University Press, August 2003., page 458, 2003.

John Canny. A computational approach to edge detection. IEEE Transactions on pattern analysis and machine intelligence, (6):679-698, 1986.

Tsung-Han Chan, Kui Jia, Shenghua Gao, Jiwen Lu, Zinan Zeng, and Yi Ma. Pcanet: A simple deep learning baseline for image classification? IEEE Transactions on Image Processing, 24(12):5017-5032, 2015.

Charles K Chui. An introduction to wavelets. Elsevier, 2016.

Nicholas P Costen, Denis M Parker, and Ian Craw. Effects of high-pass and low-pass spatial filtering on face identification. Perception \& psychophysics, 58 (4):602-612, 1996.

Ingrid Daubechies. Ten lectures on wavelets, volume 61. Siam, 1992.

Wenshuo Gao, Xiaoguang Zhang, Lei Yang, and Huizhong Liu. An improved sobel edge detection. In 2010 3rd International Conference on Computer Science and Information Technology, volume 5, pages 67-71. IEEE, 2010.

Nathan Halko, Per-Gunnar Martinsson, and Joel A Tropp. Finding structure with randomness: Probabilistic algorithms for constructing approximate matrix decompositions. SIAM review, 53(2):217-288, 2011.

Kevin Jarrett, Koray Kavukcuoglu, Marc'Aurelio Ranzato, and Yann LeCun. What is the best multi-stage architecture for object recognition? In 2009 IEEE 12th international conference on computer vision, pages 2146-2153. IEEE, 2009.

Robert A. Jarrow, Rinald Murataj, Martin T. Wells, and Liao Zhu. The low-volatility anomaly and the adaptive multi-factor model. arXiv preprint arXiv:2003.08302, 2021.

Alex Krizhevsky, Ilya Sutskever, and Geoffrey E Hinton. Imagenet classification with deep convolutional neural networks. In Advances in neural information processing systems, pages 1097-1105, 2012.

Mukesh Kumar, Rohini Saxena, et al. Algorithm and technique on various edge detection: A survey. Signal \& Image Processing, 4(3):65, 2013.

Hugo Larochelle, Dumitru Erhan, Aaron Courville, James Bergstra, and Yoshua Bengio. An empirical evaluation of deep architectures on problems with many factors of variation. In Proceedings of the 24th international conference on Machine learning, pages 473480. ACM, 2007.

Yann LeCun, Léon Bottou, Yoshua Bengio, Patrick Haffner, et al. Gradient-based learning applied to document recognition. Proceedings of the IEEE, 86(11):22782324, 1998.
Stephane Mallat. Wavelets for a vision. Proceedings of the IEEE, 84(4):604-614, 1996.

Stephane Georges Mallat. Multiresolution representations and wavelets. $\mathrm{PhD}$ thesis, University of Pennsylvania, 1988.

Klas Nordberg. Fourier transforms. 1995

Gilbert Strang. Wavelet transforms versus fourier transforms. Bulletin of the American Mathematical Society, 28(2):288-305, 1993.

Yiming Sun, Yang Guo, Charlene Luo, Joel Tropp, and Madeleine Udell. Low-rank tucker approximation of a tensor from streaming data. SIAM Journal on Mathematics of Data Science, 2(4):1123-1150, 2020.

Yiming Sun, Yang Guo, Joel A Tropp, and Madeleine Udell. Tensor random projection for low memory dimension reduction. arXiv preprint arXiv:2105.00105, 2021.

Xin Wang. Laplacian operator-based edge detectors. IEEE Transactions on Pattern Analysis and Machine Intelligence, 29(5):886-890, 2007.

Lei Yang, Xiaoyu Wu, Dewei Zhao, Hui Li, and Jun Zhai. An improved prewitt algorithm for edge detection based on noised image. In 2011 4th International Congress on Image and Signal Processing, volume 3, pages 1197-1200. IEEE, 2011.

Liao Zhu. The Adaptive Multi-Factor Model and the Financial Market. eCommons, 2020.

Liao Zhu, Sumanta Basu, Robert A. Jarrow, and Martin T. Wells. High-dimensional estimation, basis assets, and the adaptive multi-factor model. The Quarterly Journal of Finance, 10(04):2050017, 2020.

Liao Zhu, Robert A. Jarrow, and Martin T. Wells. Timeinvariance coefficients tests with the adaptive multifactor model. arXiv preprint arXiv:2011.04171, 2021a.

Liao Zhu, Ningning Sun, and Martin T. Wells. Clustering structure of microstructure measures. arXiv preprint arXiv:2107.02283, 2021b.

Liao Zhu, Haoxuan Wu, and Martin T. Wells. A newsbased machine learning model for adaptive asset pricing. arXiv preprint arXiv:2106.07103, 2021c. 